\title{
Epstein-Barr virus infection serves as an independent predictor of survival in patients with lymphoepithelioma-like gastric carcinoma
}

\author{
Byung-Hoon Min ${ }^{1}$ Chung Hyun Tae ${ }^{1} \cdot$ Soo Min $\mathrm{Ahn}^{2} \cdot$ So Young Kang ${ }^{2}$. \\ Sook-Young $\mathrm{Woo}^{3} \cdot$ Seonwoo $\mathrm{Kim}^{3} \cdot \mathrm{Kyoung}^{3}$-Mee $\mathrm{Kim}^{2}$
}

Received: 21 April 2015/Accepted: 22 July 2015/Published online: 12 August 2015

(C) The International Gastric Cancer Association and The Japanese Gastric Cancer Association 2015

\begin{abstract}
Background The pathogenesis and clinicopathologic characteristics of Epstein-Barr virus (EBV)-negative lymphoepithelioma-like gastric carcinoma (LELC) are still unclear. In addition, it remains controversial whether EBV infection itself affects the prognosis of LELC.

Methods Between 1995 and 2011, 145 LELC patients (124 patients with EBV infection and 21 patients without EBV infection) underwent radical gastrectomy with D2 lymph node dissection. The clinicopathologic features and prognosis of EBV-negative LELC cases were compared with those of EBV-positive LELC cases. The median duration of follow-up after surgery was 55 months. Microsatellite instability (MSI) analysis was performed on 20 EBV-negative LELC cases.

Results EBV-negative LELC accounted for $14.5 \%$ of the total LELC cases. EBV-negative LELC was significantly associated with older age, female sex, advanced $\mathrm{T}$ stage, and advanced American Joint Committee on Cancer (AJCC) tumor stage compared with EBV-positive LELC. In
\end{abstract}

B.-H. Min and C. H. Tae contributed equally to this work.

Kyoung-Mee Kim

kkmkys@skku.edu

Byung-Hoon Min

bhmin@skku.edu

1 Department of Medicine, Sungkyunkwan University School of Medicine, Seoul, Korea

2 Department of Pathology and Translational Genomics, Samsung Medical Center, Sungkyunkwan University School of Medicine, Irwon-dong, Gangnam-gu, Seoul 135-710, Korea

3 Biostatistics Team, Samsung Biomedical Research Institute, Seoul, Korea univariate analysis, patients with EBV-negative LELC had significantly shorter overall, disease-specific, and recurrencefree survival than those with EBV-positive LELC. The 5-year overall survival rates were $81.0 \%$ for patients with EBVnegative LELC and $96.2 \%$ for patients with EBV-positive LELC. In a Cox proportional hazards model, EBV infection, age, and AJCC tumor stage were identified as independent predictors of overall survival. MSI-high, MSI-low, and microsatellite-stable tumors accounted for 25,10 , and $65 \%$ of EBV-negative LELC cases, respectively. MSI status did not affect the prognosis of EBV-negative LELC cases.

Conclusions EBV infection serves as an independent predictor of survival in patients with LELC. EBV-negative LELC exhibited clinicopathologic features and prognosis distinct from those of EBV-positive LELC.

Keywords Lymphoepithelioma-like gastric carcinoma . Epstein-Barr virus · Prognosis

\section{Introduction}

Lymphoepithelioma-like gastric carcinoma (LELC) is a rare variant of gastric cancer (GC) and is characterized by a dense infiltration of inflammatory cells in the tumor stroma and an expansile growth pattern without desmoplasia [1]. LELC constitutes only 1-4\% of all GCs [2-4] and has been described as having a favorable prognosis compared with that of ordinary GC, especially in LELCs with Epstein-Barr virus (EBV) infection [1, 5-9]. EBV infection is found in more than $80 \%$ of LELC cases, and EBVpositive LELCs share the following features with EBVassociated GCs: male predominance, predisposition to the proximal part of the stomach, and a high proportion of diffuse-type gastric carcinomas [10-13]. 
Our previous study compared the prognosis of EBVassociated GCs according to subtypes, including LELC, GC with Crohn's disease-like lymphocytic reaction, and conventional adenocarcinoma. We reported that the prognosis of EBV-associated GCs depends on the patient's inflammatory responses $[1,14]$. Patients with EBV-positive LELC and EBV-associated GC with Crohn's disease-like lymphocytic reaction had significantly longer overall and disease-free survival than those with EBV-positive conventional adenocarcinoma. These results suggest that the degree of tumor-infiltrating immune cells may have prognostic significance in EBV-associated GC.

It remains controversial whether EBV-positive and EBV-negative GCs have different prognoses. Some studies have shown that EBV-positive GCs have prognosis comparable to that of their EBV-negative counterparts [15-17]. However, other studies have reported significantly better prognosis for EBV-positive GC than for EBV-negative GC [7, 8, 18-20]. A recent large-scale, multinational, pooled analysis showed that tumor EBV positivity was associated with lower mortality in patients with GC [12]. The more favorable prognosis of EBV-positive $\mathrm{GC}$ may be related to the lower propensity of EBV-associated GC to involve adjacent lymph nodes (LNs), perhaps resulting from immune responses directed against EBV-related proteins expressed by tumor cells [8]. In several studies, EBVpositive GCs showed more extensive infiltration of CD8 T cells and higher numbers of mature dendritic cells than EBV-negative GCs, especially in patients with EBV-positive GCs without LN metastasis [15, 16, 21-24]. These results suggest that EBV positivity itself may confer a more favorable prognosis.

A small portion of LELCs are not associated with EBV infection. These EBV-negative LELCs also show dense infiltration of immune cells in the tumor stroma similar to that of EBV-positive LELCs. As EBV-negative LELCs are very rare tumors, their pathogenesis and clinicopathologic characteristics are still unclear. In addition, it remains controversial whether EBV-negative LELCs have a prognosis distinct from that of EBV-positive LELCs [7, 25].

To address these issues, we examined the clinicopathologic features of EBV-negative LELCs and investigated whether EBV infection itself affects the prognosis of LELC.

\section{Methods}

\section{Case selection}

We searched a pathology report database including all GC cases evaluated in Samsung Medical Center between November 1995 and April 2011 using the following search terms: "lymphoepithelioma-like carcinoma," "medullary carcinoma," and "poorly differentiated carcinoma with lymphoid stroma." A total of 205 cases were identified from this search, and all these cases were reviewed by a single pathologist (K.M.K.), who is an expert in diagnosing LELC [1]. LELC was defined by (1) well-defined tumor margin, (2) dense lymphocytic infiltration of a degree whereby the number of tumor-infiltrating lymphocytes was greater than the number of tumor cells throughout the tumor, (3) indistinct cytoplasmic borders and a syncytial growth pattern with poorly formed glandular structures, and (4) no desmoplasia [1]. After a pathology review based on the strict criteria stated above, 60 cases were excluded. A total of 145 patients were finally confirmed as having LELC and enrolled in the present study. All 145 patients were treated by radical gastrectomy with $\mathrm{D} 2 \mathrm{LN}$ dissection with and without adjuvant chemotherapy. No patient had other uncontrolled cancer at the time of diagnosis of GC or during the follow-up period. Clinical data, including demographic features, tumor characteristics, and treatment outcome, were obtained by the review of medical records with use of the intranet resources of Samsung Medical Center. Tumor stage was defined according to the tumor, node, metastasis (TNM) classification (seventh edition) developed by the American Joint Committee on Cancer (AJCC) [26]. All patients provided informed consent according to our institutional guidelines. The institutional review boards at Samsung Medical Center approved the study protocol.

\section{EBV-encoded RNA in situ hybridization}

Three-micrometer-thick sections were cut from each tissue block and mounted on Superfrost-plus slides (Thermo Scientific, Waltham, MA). The entire procedure was performed with a fully automatic system (BOND-MAX) for in situ hybridization with an EBV-encoded RNA probe (Leica, Newcastle, UK) according to the manufacturer's instructions. Only sections that showed a strong signal within almost all tumor cell nuclei were considered as positive.

\section{Microsatellite instability}

Microsatellite instability (MSI) analysis was performed on EBV-negative LELC cases by multiplex polymerase chain reaction (PCR) with five quasi-monomorphic mononucleotide repeat markers as previously described [27]. Briefly, genomic DNA was isolated from formalin-fixed, paraffin-embedded tumor samples with a QIAamp DNA mini kit (Qiagen, Valencia, CA, USA) under a microscope. Each sense primer was end-labeled with one of the fluorescent markers FAM, HEX, or NED. Amplified PCR products were run on an ABI Prism 3130 Genetic Analyzer 
(Applied Biosystems, Foster City, CA, USA). Allelic sizes were estimated by Genemapper 4.1 (Applied Biosystems). Samples with no allelic size variations in any of the microsatellites were classified as microsatellite stable (MSS). Tumors with allelic size variations in one of the microsatellites were classified as MSI low, whereas those with allelic size variations in two or more of the microsatellite markers were considered to be MSI high.

\section{Statistical analysis}

Overall survival data were obtained from the national registry of medical insurance. Overall survival was measured from the date of surgery to the date of death from any cause or to the censoring date of April 30, 2014. Recurrence-free survival was measured from the date of resection to the date of the first recurrence documented by pathology review or imaging modalities, including abdominal computed tomography and ultrasonography, or to the censoring date of April 30, 2014. Overall survival and recurrence-free survival curves were estimated by Kaplan-Meier methods, and differences in survival distributions were compared with a log-rank test. Patients with stage IV gastric cancer or not undergoing follow-up examination were excluded from the calculation of recurrence-free survival. Hazard ratios and corresponding confidence intervals were estimated with a Cox proportional hazards model to identify potential predictive factors of overall survival. A $P$ value less than 0.05 was considered statistically significant.

\section{Results}

\section{Clinicopathologic characteristics according to EBV infection status}

LELC accounted for $1.0 \%(145 / 15194)$ of total GC cases treated by surgery in our institution. Of the 145 LELCs, EBV was positive in 124 cases $(85.5 \%)$ and negative in 21 cases $(14.5 \%)$. The clinicopathologic characteristics of EBV-positive and EBV-negative LELC are summarized in Table 1. EBV-negative LELC was significantly associated with older age, female sex, advanced $\mathrm{T}$ stage, and advanced tumor stage compared with EBV-positive LELC. The mean numbers of retrieved LNs during radical gastrectomy were $44.3 \pm 17.5$ for EBV-positive LELC and $43.1 \pm 11.8$ for EBV-negative LELC. The LN metastasis rates were $27.4 \%$ for EBV-positive LELC and $38.1 \%$ for EBV-negative LELC, and this difference did not reach statistical significance.

The representative pathologic findings of EBV-positive and EBV-negative LELC are depicted in Fig. 1. In
Table 1 Clinicopathologic characteristics of lymphoepithelioma-like gastric carcinoma $(L E L C)$ according to Epstein-Barr virus $(E B V)$ infection status

\begin{tabular}{|c|c|c|c|}
\hline & $\begin{array}{l}\text { EBV positive } \\
(n=124)\end{array}$ & $\begin{array}{l}\text { EBV negative } \\
(n=21)\end{array}$ & $P$ \\
\hline \multicolumn{4}{|l|}{ Age } \\
\hline Mean $\pm \mathrm{SD}$ & $56.1 \pm 9.8$ years & $62.8 \pm 12.5$ years & \multirow[t]{3}{*}{0.006} \\
\hline$<60$ years & $77(62.1 \%)$ & $9(42.9 \%)$ & \\
\hline$\geq 60$ years & $47(37.9 \%)$ & $12(57.1 \%)$ & \\
\hline \multicolumn{4}{|l|}{ Sex } \\
\hline Male & $107(86.3 \%)$ & $14(66.7 \%)$ & \multirow[t]{2}{*}{0.049} \\
\hline Female & $17(13.7 \%)$ & $7(33.3 \%)$ & \\
\hline \multicolumn{4}{|l|}{ Tumor site } \\
\hline Antrum/angle & $11(8.9 \%)$ & $4(19.0 \%)$ & \multirow[t]{2}{*}{0.235} \\
\hline Body/fundus/cardia & $113(91.1 \%)$ & $17(81.0 \%)$ & \\
\hline \multicolumn{4}{|c|}{ Depth of tumor invasion } \\
\hline $\mathrm{T} 1$ & $51(41.1 \%)$ & $2(9.5 \%)$ & \multirow[t]{4}{*}{0.040} \\
\hline $\mathrm{T} 2$ & $38(30.6 \%)$ & $11(52.4 \%)$ & \\
\hline $\mathrm{T} 3$ & $29(23.4 \%)$ & $6(28.6 \%)$ & \\
\hline $\mathrm{T} 4$ & $6(4.8 \%)$ & $2(9.5 \%)$ & \\
\hline \multicolumn{4}{|c|}{ Lymph node metastasis } \\
\hline Absent & $90(72.6 \%)$ & $13(61.9 \%)$ & \multirow[t]{2}{*}{0.319} \\
\hline Present & $34(27.4 \%)$ & $8(38.1 \%)$ & \\
\hline \multicolumn{4}{|l|}{$\mathrm{N}$ category } \\
\hline No & $90(72.6 \%)$ & $13(61.9 \%)$ & \multirow[t]{5}{*}{0.136} \\
\hline N1 (1 or 2 nodes) & $12(9.7 \%)$ & $3(14.3 \%)$ & \\
\hline N2 (3-6 nodes) & $14(11.3 \%)$ & $1(4.8 \%)$ & \\
\hline N3a (7-15 nodes) & $6(4.8 \%)$ & $4(19.0 \%)$ & \\
\hline N3b ( $\geq 16$ nodes $)$ & $2(1.6 \%)$ & $0(0.0 \%)$ & \\
\hline \multicolumn{4}{|l|}{ AJCC stage } \\
\hline I & $80(64.5 \%)$ & $8(38.1 \%)$ & \multirow[t]{4}{*}{0.009} \\
\hline II & $27(21.8 \%)$ & $11(52.4 \%)$ & \\
\hline III & $16(12.9 \%)$ & $1(4.8 \%)$ & \\
\hline IV & $1(0.8 \%)$ & $1(4.8 \%)$ & \\
\hline \multicolumn{4}{|c|}{ Eosinophils and neutrophils admixed with lymphoplasma cells } \\
\hline Absent & $120(96.8 \%)$ & $16(76.2 \%)$ & \multirow[t]{2}{*}{0.003} \\
\hline Present & $4(3.2 \%)$ & $5(23.8 \%)$ & \\
\hline \multicolumn{4}{|l|}{ Non-LELC component } \\
\hline Absent & $34(27.4 \%)$ & $5(23.8 \%)$ & \multirow[t]{2}{*}{0.730} \\
\hline Present & $90(72.6 \%)$ & $16(76.2 \%)$ & \\
\hline \multicolumn{4}{|c|}{ Adjuvant chemotherapy } \\
\hline No & $84(67.7 \%)$ & $11(52.4 \%)$ & \multirow[t]{2}{*}{0.171} \\
\hline Yes & $40(32.3 \%)$ & $10(47.6 \%)$ & \\
\hline
\end{tabular}

AJCC American Joint Committee on Cancer, SD standard deviation

pathology review, EBV-negative LELCs showed some eosinophils and neutrophils admixed with lymphoplasma cells in $23.8 \%$ of cases. However, EBV-positive LELCs demonstrated such features in only $3.2 \%$ of cases, which was significantly lower than that of EBV-negative LELCs (Fig. 2). A non-LELC component, such as tubular 

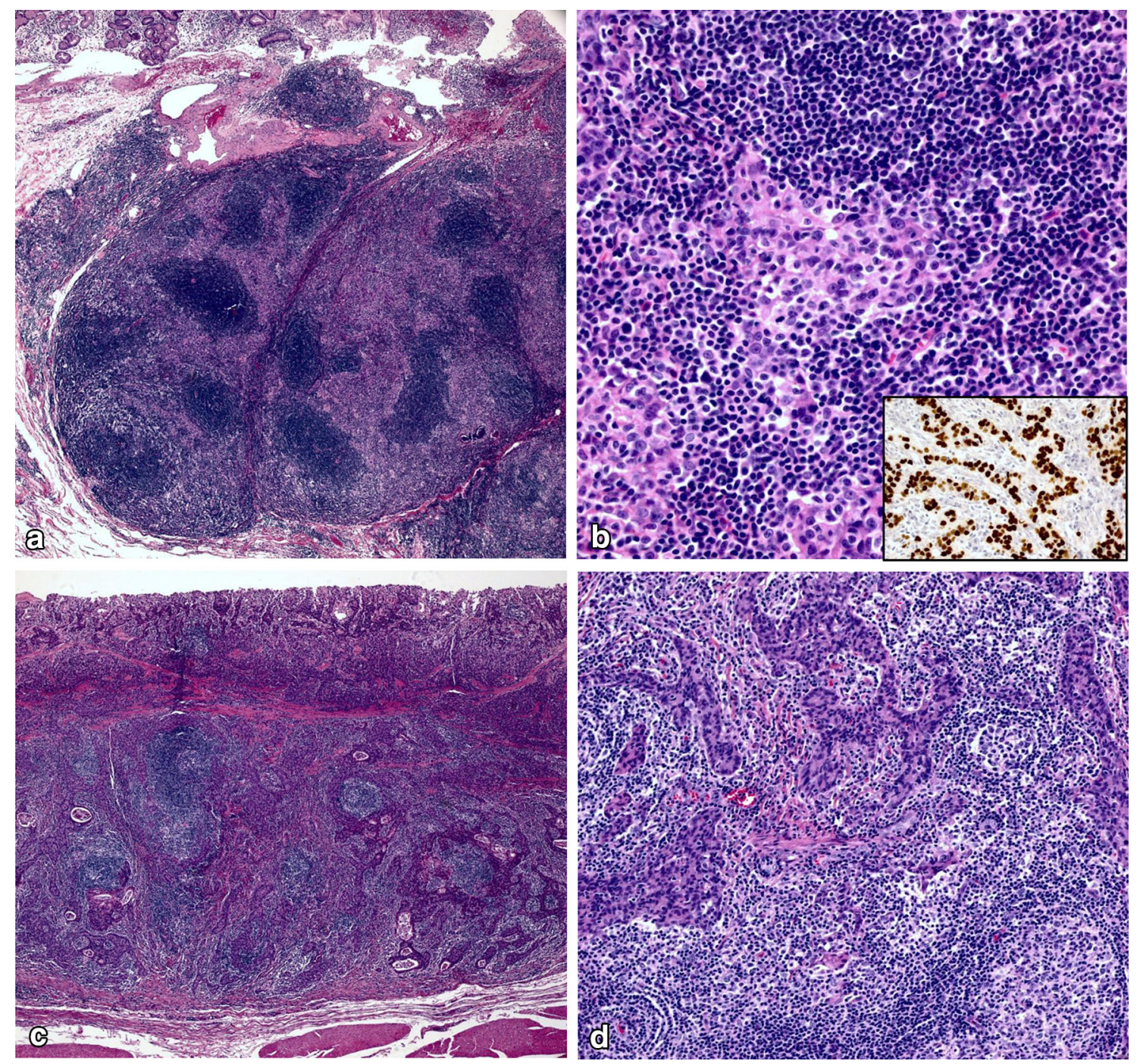

Fig. 1 Representative pathologic findings of lymphoepithelioma-like gastric carcinoma (LELC). a, b Epstein-Barr virus (EBV)-positive LELC with prominent lymphoid follicles and expanding growth pattern $(\mathbf{a} \times 5, \mathbf{b} \times 40$, inset $\mathrm{EBV}$ in situ hybridization, hematoxylin

and eosin stain). c, d Microsatellite-instability-high EBV-negative LELC with prominent lymphoid stroma and expanding growth pattern (c $\times 5, \mathbf{d} \times 40$, hematoxylin and eosin stain). Note that a non-LELC component is present in the mucosal area in the surface

adenocarcinoma, was observed in 72.6 and $76.2 \%$ of EBV-positive and EBV-negative LELCs, respectively. The mean percentages of the non-LELC component were $13.0 \%$ (range 0-60\%) in EBV-positive LELCs and $15.5 \%$ (range 0-60 \%) in EBV-negative LELCs, and were comparable between the two groups. The non-LELC component was usually located in the mucosal or surface portion of the tumors.

\section{Impact of EBV infection on prognosis}

To address the prognostic significance of EBV infection in LELC, we analyzed overall and recurrence-free survival.
The median duration of follow-up was 55 months (range 3-216 months) for overall survival analysis and 48 months (range 2-171 months) for recurrence-free survival analysis. In univariate analysis, patients with EBV-negative LELC showed significantly shorter overall and recurrence-free survival compared with those with EBV-positive LELC (Table 2, Fig. 3). The 5-year overall survival rates were $81.0 \%$ for patients with EBV-negative LELC and $96.2 \%$ for patients with EBV-positive LELC. Disease-specific survival was also significantly worse in patients with EBVnegative LELC. The 5-year disease-specific survival rates were $90.5 \%$ for patients with EBV-negative LELC and 99.2 \% for patients with EBV-positive LELC (Fig. 3). In a 


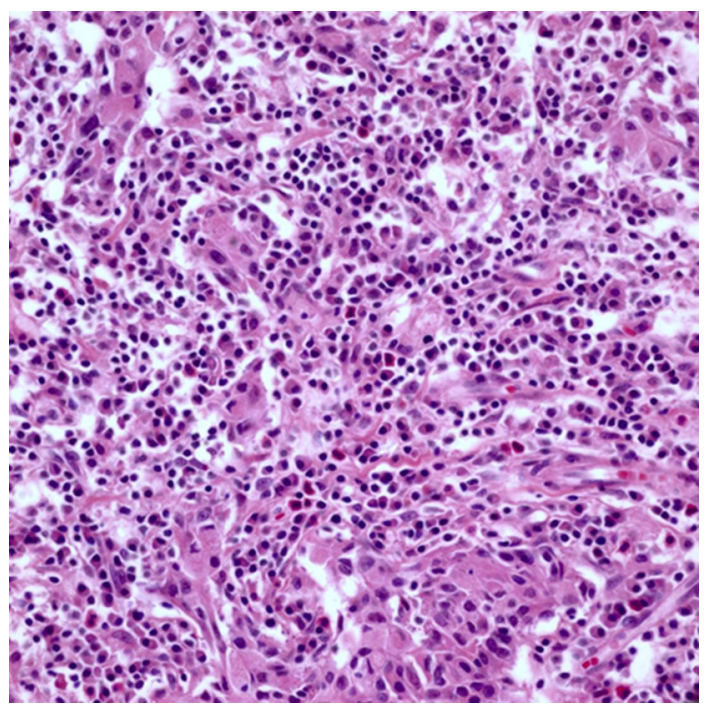

Fig. 2 Pathology of Epstein-Barr virus negative lymphoepitheliomalike gastric carcinoma showing some eosinophils and neutrophils admixed with many lymphoplasma cells $(\times 200$, hematoxylin and eosin stain)

Cox proportional hazards model with adjustment for age, sex, tumor site, and AJCC stage, EBV infection was identified as an independent predictor of overall survival (Table 3). The estimated mortality risk of EBV-negative LELC patients was five times as high as that of EBVpositive LELC patients. Age and AJCC stage were also identified as independent predictors of overall survival. As the model was not fitted, multivariate analysis of recurrence-free or disease-specific survival could not be performed.

\section{MSI in EBV-negative LELCs}

MSI analysis was successfully performed in $20 \mathrm{EBV}$-negative LELC cases with five quasi-monomorphic mononucleotide repeat markers. Five cases $(25 \%)$ among EBVnegative LELCs were MSI high, two (10\%) were MSI low, and $13(65 \%)$ were MSS. MSI-high EBV-negative LELC cases were significantly associated with older age compared with MSS or MSI-low EBV-negative LELC cases (74.4 \pm 3.6 years for MSI-high cases and $59.1 \pm 12.6$ years for MSS or MSI-low cases). However, no significant differences were observed between the two groups for sex, tumor site, depth of tumor invasion, presence of LN metastasis, or AJCC tumor stage. Overall survival and recurrence-free survival of MSI-high cases showed no significant difference from those of MSS or MSI-low cases.

\section{Discussion}

In the present study, we compared the clinicopathologic features and prognosis of $124 \mathrm{EBV}$-positive LELCs and 21 EBV-negative LELCs. EBV-negative LELC was

Table 2 Univariate analysis of overall survival $(O S)$ and recurrence-free survival $(R F S)$ in patients with lymphoepithelioma-like gastric carcinoma

\begin{tabular}{|c|c|c|c|c|c|c|}
\hline & \multicolumn{3}{|l|}{ Overall survival } & \multicolumn{3}{|c|}{ Recurrence-free survival ${ }^{\mathrm{a}}$} \\
\hline & 3 -year OS rate $(\%)$ & 5 -year OS rate $(\%)$ & $P$ & 3-year RFS rate $(\%)$ & 5-year RFS rate $(\%)$ & $P$ \\
\hline \multicolumn{7}{|l|}{ Age (years) } \\
\hline$<60$ & 97.7 & 96.0 & \multirow[t]{2}{*}{0.068} & 97.5 & 97.5 & \multirow[t]{2}{*}{0.183} \\
\hline$\geq 60$ & 93.2 & 91.1 & & 100.0 & 100.0 & \\
\hline \multicolumn{7}{|l|}{ Sex } \\
\hline Male & 95.0 & 92.7 & \multirow[t]{2}{*}{0.128} & 98.1 & 98.1 & \multirow[t]{2}{*}{0.431} \\
\hline Female & 100.0 & 100.0 & & 100.0 & 100.0 & \\
\hline \multicolumn{7}{|l|}{ EBV } \\
\hline Positive & 98.4 & 96.2 & \multirow[t]{2}{*}{0.004} & 99.1 & 99.1 & \multirow[t]{2}{*}{0.007} \\
\hline Negative & 81.0 & 81.0 & & 94.7 & 94.7 & \\
\hline \multicolumn{7}{|l|}{ Tumor site } \\
\hline Antrum/angle & 100.0 & 100.0 & \multirow[t]{2}{*}{0.663} & 100.0 & 100.0 & \multirow[t]{2}{*}{0.155} \\
\hline Body/fundus/cardia & 95.4 & 93.4 & & 98.3 & 98.3 & \\
\hline \multicolumn{7}{|l|}{ AJCC stage } \\
\hline I & 97.7 & 95.9 & \multirow[t]{3}{*}{0.024} & 100.0 & 100.0 & \multirow[t]{3}{*}{0.014} \\
\hline II & 97.4 & 94.6 & & 100.0 & 100.0 & \\
\hline III-IV & 84.2 & 84.2 & & 87.5 & 87.5 & \\
\hline
\end{tabular}

AJCC American Joint Committee on Cancer, EBV Epstein-Barr virus

${ }^{a}$ Patients with stage IV gastric cancer or not undergoing follow-up examination were excluded from the calculation of RFS 

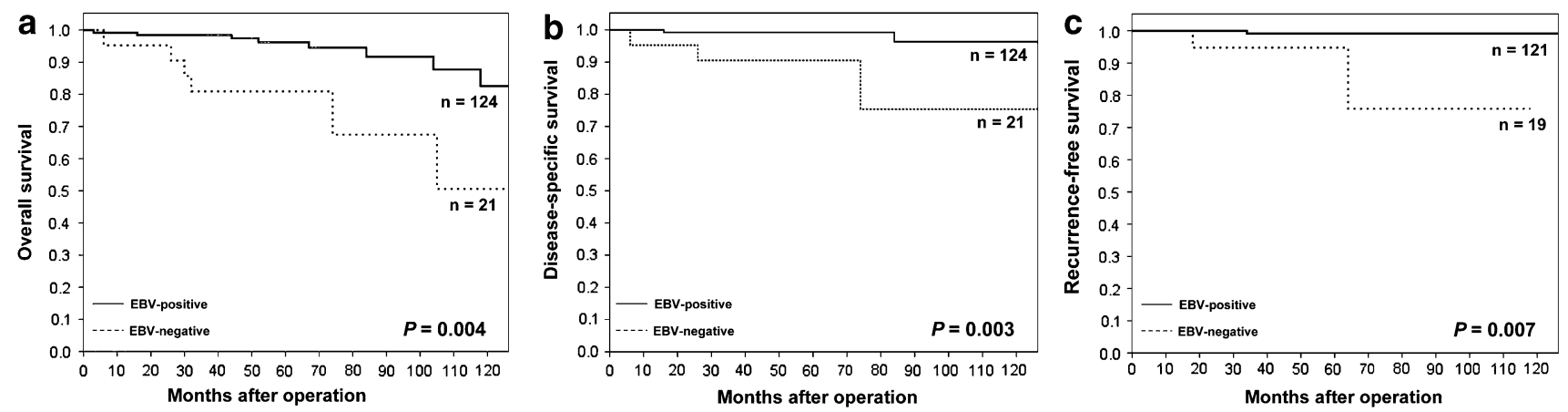

Fig. 3 Overall, disease-specific, and recurrence-free survival of patients with lymphoepithelioma-like gastric carcinoma according to the Epstein-Barr virus $(E B V)$ infection status: a Kaplan-Meier

curves for overall survival: b Kaplan-Meier curves for diseasespecific survival; c Kaplan-Meier curves for recurrence-free survival

Table 3 Multivariate analysis of overall survival in patients with lymphoepithelioma-like gastric carcinoma

\begin{tabular}{llll}
\hline & \multicolumn{3}{l}{ Cox proportional hazards model } \\
\cline { 2 - 4 } & Hazard ratio & $95 \%$ CI & $P$ \\
\hline Age & 1.110 & $1.040-1.185$ & 0.002 \\
Sex & & & \\
Male & 1 (reference) & & 0.065 \\
Female & 0.111 & $0.011-1.148$ & \\
EBV & & & \\
Positive & 1 (reference) & & \\
Negative & 5.151 & $1.398-18.984$ & 0.014 \\
Tumor site & & & \\
Antrum/angle & 1 (reference) & & \\
Body/fundus/cardia & 0.695 & $0.066-7.310$ & 0.762 \\
AJCC stage & & & \\
I & 1 (reference) & & $0.816^{*}$ \\
II & 0.510 & $0.104-2.508$ & $0.002^{*}$ \\
III-IV & 9.406 & $2.359-37.507$ &
\end{tabular}

AJCC American Joint Committee on Cancer, $C I$ confidence interval, $E B V$ Epstein-Barr virus

* $P$ values were corrected by Bonferroni's method because of multiple testing

significantly associated with older age, female sex, advanced $\mathrm{T}$ stage, and advanced AJCC tumor stage compared with EBV-positive LELC. In addition, patients with EBV-negative LELC had significantly shorter overall, disease-specific, and recurrence-free survival than those with EBV-positive LELC. EBV infection was an independent prognostic factor in patients with LELC.

There have been a few small studies addressing the features of EBV-negative LELC. Chang et al. [25] found that EBV-negative GCs with lymphoid stroma were significantly associated with female sex and distal tumor location compared with EBV-positive tumors. Nakamura et al. [7] also reported similar findings. In addition, they found EBV-positive GCs with lymphoid stroma were significantly more frequently associated with lymphoid infiltration within the cancer cell nest and a marked degree of lymphoid stroma compared with their EBV-negative counterparts. In their study, EBV-positive tumors also showed a significantly lower mitotic rate than EBV-negative tumors. These histologic features of EBV-positive LELC might have an effect on prognosis as several studies reported that an increased tumor-infiltrating lymphocyte count is significantly associated with a favorable survival in GCs [5]. The extensive infiltration of tumor nests with cytotoxic CD8 lymphocytes may promote eradication of EBV-positive malignant cells $[22,28]$. In the present study, patients with EBV-positive LELC had significantly longer overall, disease-specific, and recurrence-free survival compared with those without EBV infection, and EBV infection itself was identified as an independent predictor of overall survival. The above-mentioned histologic features of EBV-associated LELC such as lymphoid infiltration within the cancer cell nest count could plausibly explain the favorable survival of patients with EBV-positive LELC that we observed in the present study. In the present study, both EBV-positive and EBV-negative LELCs had extensive lymphoid stroma in all cases by definition, and we could not classify LELCs by the degree of lymphoid stroma.

Two subsets of GC, EBV-positive and MSI-high cancers, have been associated with abundant lymphocytic infiltration in the tumor stroma, a characteristic finding of LELC [9, 29]. More than $80 \%$ of LELCs are related to EBV infection, whereas a small subset is associated with MSI [9]. Several studies reported cases of EBV-negative LELCs with an MSI-high phenotype [5, 30]. There is persuasive evidence that EBV infection and MSI are mutually exclusive, especially in GCs with a lymphoid stroma [5, 9, 31, 32]. Therefore, there is high probability that carcinogenesis of EBV-negative LELCs is associated with MSI. In this first study with a large number of EBV- 
negative LELC cases undergoing MSI examination, we found that MSI-high tumors accounted for $25 \%$ of EBVnegative cases. MSI status did not affect the prognosis of EBV-negative LELC cases. Further studies are required to identify the pathogenesis of EBV-negative LELCs.

This study was limited in that it was performed at a single tertiary referral center and had a retrospective design. As LELCs, especially EBV-negative LELCs, are very rare tumors, prospective collection of a sufficient number of samples would require quite a long time and thus is practically impossible. The present study had several strengths. First, LELC usually has a favorable prognosis, and recurrence after surgery is very rare. Therefore, a large sample size and long follow-up duration are definitely required for an adequate evaluation of survival of patients with LELC. To the best of our knowledge, this study included the largest number of LELC cases ever reported, and the median follow-up duration was 55 months, which enabled us to reliably assess and compare long-term survival between EBV-positive and EBV-negative LELC patients. Second, this study included a large number of EBV-negative LELC patients, and MSI status was examined in most cases. This contributed to our evaluation of the role of MSI in the pathogenesis and features of EBVnegative LELC.

In conclusion, EBV infection serves as an independent predictor of survival in patients with LELC. EBV-negative LELC exhibited clinicopathologic features and prognosis distinct from those of EBV-positive LELC.

Acknowledgments This research was supported by a grant from the 20 by 20 project of Samsung Medical Center (GF01140111).

\section{Compliance with ethical standards}

Conflict of interest The authors declare that they have no conflict of interest.

\section{References}

1. Song HJ, Srivastava A, Lee J, Kim YS, Kim KM, Ki Kang W, et al. Host inflammatory response predicts survival of patients with Epstein-Barr virus-associated gastric carcinoma. Gastroenterology. 2010;139(84-92):e2.

2. Corvalan A, Ding S, Koriyama C, Carrascal E, Carrasquilla G, Backhouse C, et al. Association of a distinctive strain of EpsteinBarr virus with gastric cancer. Int J Cancer. 2006;118:1736-42.

3. Burke AP, Yen TS, Shekitka KM, Sobin LH. Lymphoepithelial carcinoma of the stomach with Epstein-Barr virus demonstrated by polymerase chain reaction. Mod Pathol. 1990;3:377-80.

4. Horiuchi K, Mishima K, Ohsawa M, Aozasa K. Carcinoma of stomach and breast with lymphoid stroma: localisation of Epstein-Barr virus. J Clin Pathol. 1994;47:538-40.

5. Grogg KL, Lohse CM, Pankratz VS, Halling KC, Smyrk TC. Lymphocyte-rich gastric cancer: associations with Epstein-Barr virus, microsatellite instability, histology, and survival. Mod Pathol. 2003;16:641-51.

6. Kijima Y, Ishigami S, Hokita S, Koriyama C, Akiba S, Eizuru Y, et al. The comparison of the prognosis between Epstein-Barr virus (EBV)-positive gastric carcinomas and $\mathrm{EBV}$-negative ones. Cancer Lett. 2003;200:33-40.

7. Nakamura S, Ueki T, Yao T, Ueyama T, Tsuneyoshi M. EpsteinBarr virus in gastric carcinoma with lymphoid stroma. Special reference to its detection by the polymerase chain reaction and in situ hybridization in 99 tumors, including a morphologic analysis. Cancer. 1994;73:2239-49.

8. van Beek J, Zur Hausen A, Klein Kranenbarg E, van de Velde CJ, Middeldorp JM, van den Brule AJ, et al. EBV-positive gastric adenocarcinomas: a distinct clinicopathologic entity with a low frequency of lymph node involvement. J Clin Oncol. 2004;22:664-70.

9. Herath $\mathrm{CH}$, Chetty R. Epstein-Barr virus-associated lymphoepithelioma-like gastric carcinoma. Arch Pathol Lab Med. 2008;132:706-9.

10. Lee JH, Kim SH, Han SH, An JS, Lee ES, Kim YS. Clinicopathological and molecular characteristics of Epstein-Barr virusassociated gastric carcinoma: a meta-analysis. J Gastroenterol Hepatol. 2009;24:354-65.

11. Murphy G, Pfeiffer R, Camargo MC, Rabkin CS. Meta-analysis shows that prevalence of Epstein-Barr virus-positive gastric cancer differs based on sex and anatomic location. Gastroenterology. 2009;137:824-33.

12. Camargo MC, Kim WH, Chiaravalli AM, Kim KM, Corvalan $\mathrm{AH}$, Matsuo $\mathrm{K}$, et al. Improved survival of gastric cancer with tumour Epstein-Barr virus positivity: an international pooled analysis. Gut. 2014;63:236-43.

13. Park S, Choi MG, Kim KM, Kim HS, Jung SH, Lee JH, et al. Lymphoepithelioma-like carcinoma: a distinct type of gastric cancer. J Surg Res. 2015;194:458-63.

14. Song HJ, Kim KM. Pathology of epstein-barr virus-associated gastric carcinoma and its relationship to prognosis. Gut Liver. 2011;5:143-8.

15. Chang MS, Lee HS, Kim CW, Kim YI, Kim WH. Clinicopathologic characteristics of Epstein-Barr virus-incorporated gastric cancers in Korea. Pathol Res Pract. 2001;197:395-400.

16. Gulley ML, Pulitzer DR, Eagan PA, Schneider BG. Epstein-Barr virus infection is an early event in gastric carcinogenesis and is independent of bcl-2 expression and p53 accumulation. Hum Pathol. 1996;27:20-7.

17. Park ES, Do IG, Park CK, Kang WK, Noh JH, Sohn TS, et al. Cyclooxygenase-2 is an independent prognostic factor in gastric carcinoma patients receiving adjuvant chemotherapy and is not associated with EBV infection. Clin Cancer Res. 2009;15:291-8.

18. Lertprasertsuke N, Tsutsumi Y. Gastric carcinoma with lymphoid stroma. Analysis using mucin histochemistry and immunohistochemistry. Virchows Arch A Pathol Anat Histopathol. 1989;414:231-41.

19. Minamoto T, Mai M, Watanabe K, Ooi A, Kitamura T, Takahashi Y, et al. Medullary carcinoma with lymphocytic infiltration of the stomach. Clinicopathologic study of 27 cases and immunohistochemical analysis of the subpopulations of infiltrating lymphocytes in the tumor. Cancer. 1990;66:945-52.

20. Watanabe H, Enjoji M, Imai T. Gastric carcinoma with lymphoid stroma. Its morphologic characteristics and prognostic correlations. Cancer. 1976;38:232-43.

21. Lee HS, Chang MS, Yang HK, Lee BL, Kim WH. Epstein-Barr virus-positive gastric carcinoma has a distinct protein expression profile in comparison with Epstein-Barr virus-negative carcinoma. Clin Cancer Res. 2004;10:1698-705. 
22. Saiki Y, Ohtani H, Naito $Y$, Miyazawa M, Nagura $H$. Immunophenotypic characterization of Epstein-Barr virus-associated gastric carcinoma: massive infiltration by proliferating CD8+ T-lymphocytes. Lab Invest. 1996;75:67-76.

23. Tokunaga M, Land CE, Uemura Y, Tokudome T, Tanaka S, Sato E. Epstein-Barr virus in gastric carcinoma. Am $\mathrm{J}$ Pathol. 1993;143:1250-4.

24. van Beek J, Zur Hausen A, Snel SN, Berkhof J, Kranenbarg EK, van de Velde CJ, et al. Morphological evidence of an activated cytotoxic T-cell infiltrate in EBV-positive gastric carcinoma preventing lymph node metastases. Am J Surg Pathol. 2006;30:59-65.

25. Chang MS, Kim WH, Kim CW, Kim YI. Epstein-Barr virus in gastric carcinomas with lymphoid stroma. Histopathology. 2000;37:309-15.

26. American Joint Committee on Cancer. AJCC cancer staging manual. 7th ed. New York: Springer; 2010.

27. Lee SE, Kang SY, Cho J, Lee B, Chang DK, Woo H, et al. Pyloric gland adenoma in Lynch syndrome. Am J Surg Pathol. 2014;38:784-92.

28. Kuzushima K, Nakamura S, Nakamura T, Yamamura Y, Yokoyama N, Fujita M, et al. Increased frequency of antigen- specific $\mathrm{CD}^{+}$cytotoxic $\mathrm{T}$ lymphocytes infiltrating an EpsteinBarr virus-associated gastric carcinoma. $\mathrm{J}$ Clin Invest. 1999;104:163-71.

29. Chiaravalli AM, Feltri M, Bertolini V, Bagnoli E, Furlan D, Cerutti $\mathrm{R}$, et al. Intratumour $\mathrm{T}$ cells, their activation status and survival in gastric carcinomas characterised for microsatellite instability and Epstein-Barr virus infection. Virchows Arch. 2006;448:344-53.

30. Leung SY, Yuen ST, Chung LP, Chu KM, Wong MP, Branicki FJ, et al. Microsatellite instability, Epstein-Barr virus, mutation of type II transforming growth factor beta receptor and BAX in gastric carcinomas in Hong Kong Chinese. $\mathrm{Br} \mathrm{J}$ Cancer. 1999;79:582-8.

31. Chetty R. Gastrointestinal cancers accompanied by a dense lymphoid component: an overview with special reference to gastric and colonic medullary and lymphoepithelioma-like carcinomas. J Clin Pathol. 2012;65:1062-5.

32. Park HY, Kang SY, Kang GH, Bae GE, Lee SE, Kim KM, et al. EBV infection and mismatch repair deficiency mediated by loss of hMLH1 expression contribute independently to the development of multiple synchronous gastric carcinomas. J Surg Oncol. 2012;106:777-82. 